УДК 544.344

\title{
Bilayer Magnetic Structures with Dipolar Interaction in Magnetic Field
}

\author{
Anna P. Soldusova* \\ Pavel V. Prudnikov ${ }^{\dagger}$ \\ Omsk State University \\ Mira, 55a, Omsk, 644077 \\ Russia
}

Received 10.08.2016, received in revised form 10.10.2016, accepted 14.12.2016

Monte Carlo simulation was conducted for bilayer magnetic structures with dipolar interlayer interaction in an external magnetic field. Specific hysteresis loops were obtained for the system. Non-equilibrium relaxation of the autocorrelation function was considered with the high-and low-temperature initial states

Keywords: bilayer magnetic structure, dipolar interaction, hysteresis.

DOI: 10.17516/1997-1397-2017-10-2-257-260.

\section{Introduction}

Multilayer magnetic structures, consisting of magnetic and non-magnetic layers, are applied in electronic and spintronic devices [1-2], like storage and high-density record devices [3] and high-sensitive magnetic field sensors [4]. The layer magnetization can be controlled by an external applied magnetic field. Investigation of multilayer structures behaviour in the magnetic field can be carried out by a computer simulation. This work deals with structures consisting of two magnetic layers with dipolar interlayer interaction.

\section{Model and simulation}

Monte Carlo simulation was conducted for studying behaviour of bilayer structures in the magnetic field. The system Hamiltonian $H=H_{1}+H_{2}+H_{12} . \quad H_{\alpha=1,2}$ is the Hamiltonian of the first or the second magnetic layers, $H_{12}$ describes interaction between the layers. The Hamiltonians have the following form

$$
H_{\alpha}=-J \sum_{<i, j>} \mathbf{S}_{i} \cdot \mathbf{S}_{j}-A \sum_{i} S_{i, z}^{2}-\mathbf{h} \sum_{i} \mathbf{S}_{i}, \quad H_{12}=D \sum_{i j}\left(\frac{\mathbf{S}_{i} \cdot \mathbf{S}_{j}}{r_{i j}^{3}}-3 \frac{\left(\mathbf{S}_{i} \cdot \mathbf{r}_{i j}\right)\left(\mathbf{S}_{j} \cdot \mathbf{r}_{i j}\right)}{r_{i j}^{5}}\right),
$$

where $\mathbf{S}_{i}$ is a spin (a unit vector in a three-dimensional space) situated in the i-th site of the simple cubic lattice with the linear size $\mathrm{L}$ in $\mathrm{X}$ - and $\mathrm{Y}$-directions, and with the linear size $\mathrm{N}$ in Z-direction. The value of $\mathrm{N}$ corresponds to the thickness of the layer. The first term in $H_{\alpha}$ describes the exchange interaction between neighbouring spins. The second term describes the uniaxial anisotropy, with Z-direction being an easy magnetization axis provided that $A>0$. The third term describes interaction of the spins with the external magnetic field $\mathbf{h}$. $H_{12}$ has a form of dipolar interaction, summation conducted for all pairs of spins, with one spin belonging to the first layer and the other spin to the second one. In this work the Hamiltonian parameters were

\footnotetext{
*anka_omsk@mail.ru

†prudnikp@univer.omsk.su

(c) Siberian Federal University. All rights reserved
} 
set to be $J=1.00, A=0.10, D=0.01$. The system with magnetic layer thickness $N=1$ and a distance between layers $S=1$ was considered. The external magnetic field was perpendicular to the surface of the layers (parallel to Z-direction).

\section{Equilibrium behaviour of bilayer structures in magnetic field}

Characteristics of the system as a whole are the total magnetization $\mathbf{M}=\mathbf{M}_{1}+\mathbf{M}_{2}$ and the staggered magnetization $\mathbf{M}_{s t g}=\mathbf{M}_{\mathbf{1}}-\mathbf{M}_{\mathbf{2}}$, where $\mathbf{M}_{\mathbf{1}}\left(\mathbf{M}_{\mathbf{2}}\right)$ is the first (second) magnetic layer magnetization, which is a sum of spins in the layer. Lowercase letters denote values per spin.

The ground state of the system without an external magnetic field is that with antiparallel orientation of the layer magnetization vectors. This means that at the ground state $m=0.0$ and $m_{s t g}=1.0$. The same picture remains for the structure in a weak magnetic field. A strong enough field can lead all spins to overturn in the field direction, so that at the ground state $m=1.0$ and $m_{\text {stg }}=0.0$. Temperature dependence of the total and staggered magnetization is demonstrated in Fig. 1 for the weak $(h=0.05)$ and strong $(h=0.10)$ magnetic fields. A medium field was found to be $h \approx 0.07$.
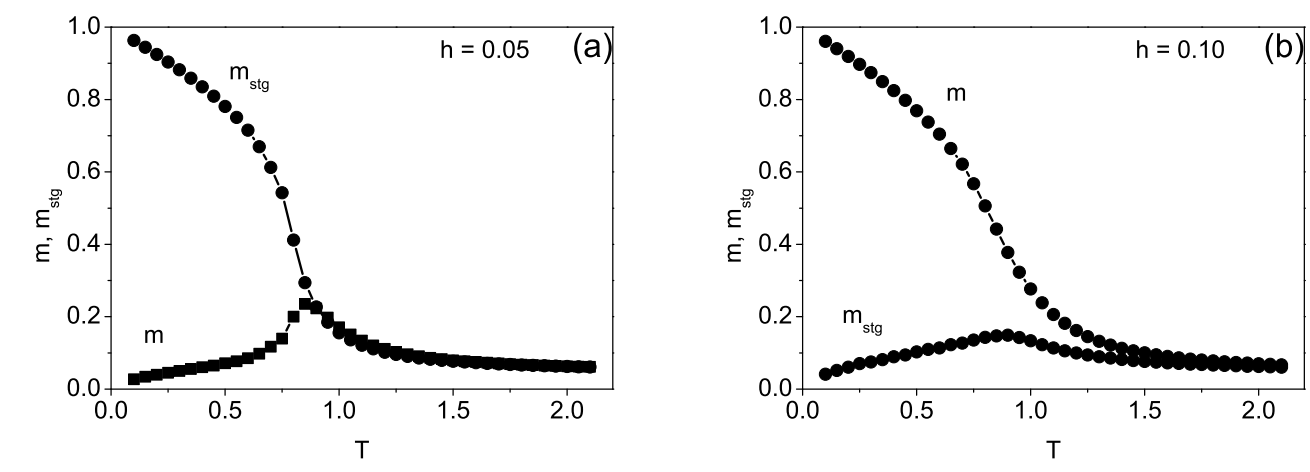

Fig. 1. Temperature dependence of the total and staggered magnetization in (a): the weak field $h=0.05,(\mathbf{b})$ : the strong field $h=0.10$

Simulation conducted in a slowly changing magnetic field reveals specific hysteresis loops for the system. Fig. 2(a) shows such loops formed by Z-projections of the total magnetization at a low temperature $T=0.1$. Two specific regions at positive and negative fields with magnitude $h=0.1-0.3$ correspond to the antiparallel state. The higher the temperature the closer are these regions to the point with $h=0.0$, until at the temperature $T \approx 0.4$ (Fig. 2(b)) they merge. Further increasing of the temperature leads to decreasing of hysteresis loops square until hysteresis disappears.

\section{Non-equilibrium behaviour of bilayer structures in magnetic field}

In the non-equilibrium simulation the autocorrelation function was calculated $C\left(t, t_{w}\right)=$ $\left\langle\frac{1}{N_{s}} \sum_{i=1}^{N_{s}} \mathbf{S}_{i}(t) \mathbf{S}_{i}\left(t_{w}\right)\right\rangle-\left\langle\frac{1}{N_{s}} M(t)\right\rangle\left\langle\frac{1}{N_{s}} M\left(t_{w}\right)\right\rangle$, where $t_{w}<t$ is a waiting time. Fig. 3 shows 

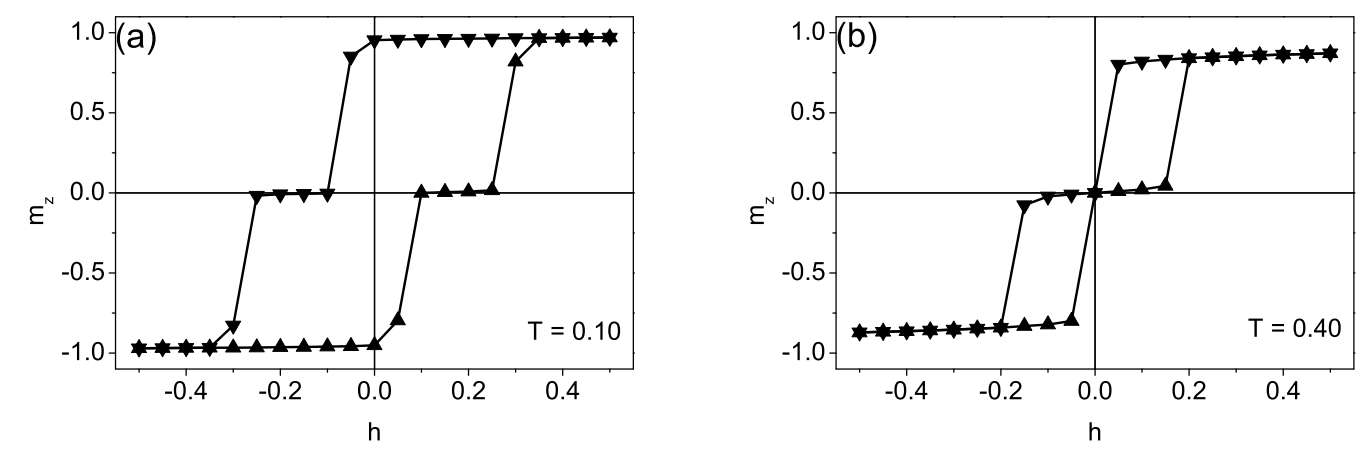

Fig. 2. Dependence of a Z-projection of the total magnetization at the temperature (a): $T=0.1$, (b): $T=0.4$. $\mathbf{\Delta}$ - the magnetic field is changed from negative to positive values, $\mathbf{\nabla}$ - vice versa

time dependence of the autocorrelation function with the high-temperature initial state $\left(m^{0} \ll\right.$ $\left.1.0, m_{s t g}^{0} \ll 1.0\right)$. The greater the waiting time the slower is relaxation of the autocorrelation function. The simulation started with the low-temperature initial state $\left(m^{0}=0.0, m_{\text {stg }}^{0}=1.0\right)$ demonstrates inverse behaviour of the autocorrelation function with the waiting time (Fig. 4). The relaxation process in magnetic field continues for longer time and up to smaller values in both cases, but for the low-temperature initial state this is more profound.
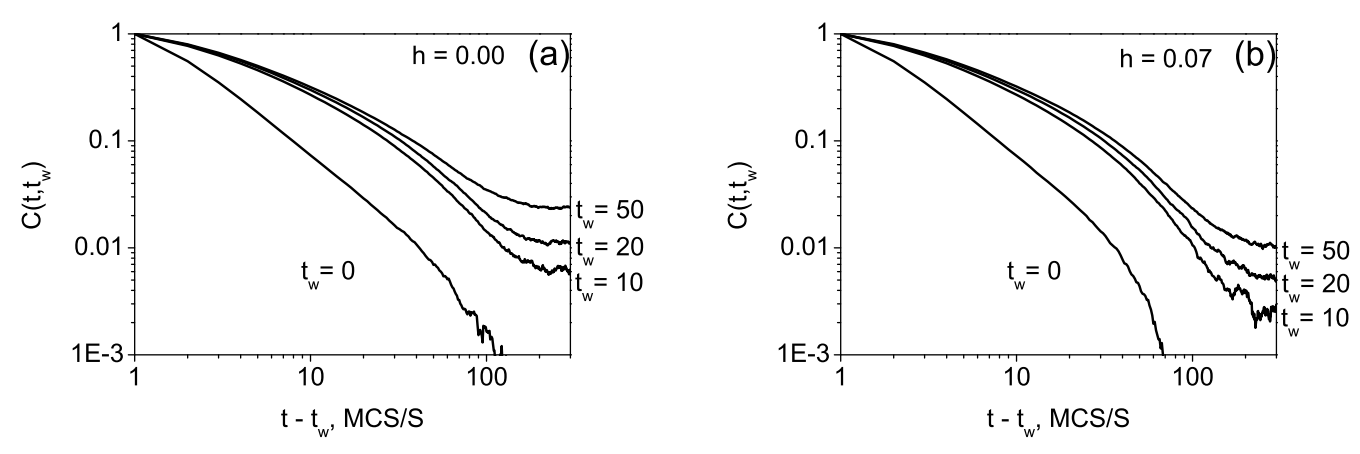

Fig. 3. Time dependence of the autocorrelation function for different waiting times with the high-temperature initial state at the temperature $T=0.9(\mathbf{a})$ : without the magnetic field, $(\mathbf{b})$ : in the magnetic field $h=0.07$

\section{Conclusions}

Simulation of bilayer structures with dipolar interaction demonstrated, that the hysteresis loops had regions with antiparallel orientation of the layer magnetization vectors. Relaxation processes of the autocorrelation function in the magnetic field took longer and continued up to smaller values, than when simulation was carried out without the magnetic field.

This work was supported by Russian Science Foundation (project no. 14-12-00562). The simulations were supported by the Supercomputing Center of Lomonosov Moscow State University, 

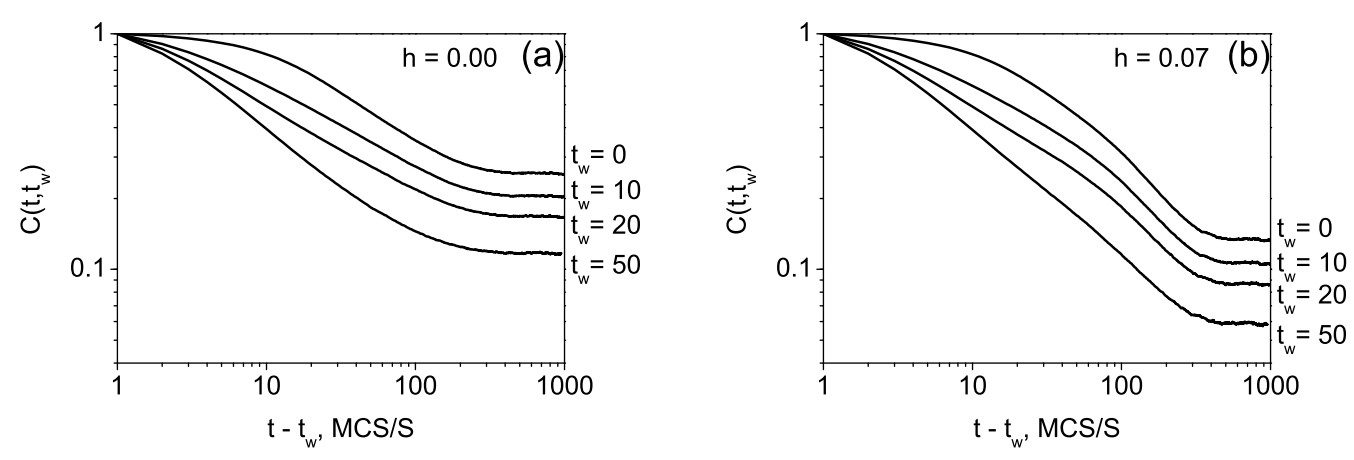

Fig. 4. Time dependence of the autocorrelation function for different waiting times with the low-temperature initial state at the temperature $T=0.9(\mathbf{a})$ : without the magnetic field, $(\mathbf{b})$ : in the magnetic field $h=0.07$

Moscow and St. Petersburg Joint Supercomputer Center of the Russian Academy of Sciences.

\section{References}

[1] C.Chappert, A.Fert, F.Nguyen Van Dau, The emergence of spin electronics in data storage, Nat. mater., 6(2007), 813-823.

[2] Žutić I., Fabian J., Das Sarma S, Spintronics: Fundamentals and applications, Rev. Mod. Phys. 76(2004), 323-410.

[3] B.Heinrich, J.A.C.Bland, Eds., Ultrathin Magnetic Structures IV, Berlin, Springer, 2005.

[4] M.Melzer, M.Kaltenbrunner, D.Makarov, D.Karnaushenko, D.Karnaushenko, T.Sekitani, T.Someya, O.G.Schmidt, Imperceptible magnetoelectronics, Nat. Commun. 6(2015), 6080.

\section{Двухслойные магнитные структуры с диполь-дипольным взаимодействием в магнитном поле}

Анна П. Солдусова

Павел В. Прудников

Омский государственный университет Мира, 55а, Омск, 644077

Россия

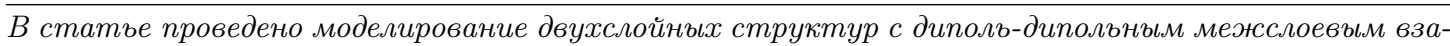
имодействием во внешнем магнитном поле. Получены специфические петли гистерезиса. Рассмотрено неравновесное поведение структур в поле и без поля при моделировании из высоко- и низкотемпературных начальных состояний.

Ключевые слова: двухслойные структуръ, диполь-дипольное взаимодействие, гистерезис. 